\title{
Relativistic Milne-Eddington Type Solutions with a Variable Eddington Factor for Relativistic Spherical Winds
}

\author{
Jun Fukue \\ Astronomical Institute, Osaka Kyoiku University, Asahigaoka, Kashiwara, Osaka 582-8582, Japan \\ Correspondence should be addressed to Jun Fukue, fukue@cc.osaka-kyoiku.ac.jp
}

Received 22 December 2010; Accepted 19 March 2011

Academic Editor: Jerome Orosz

Copyright () 2011 Jun Fukue. This is an open access article distributed under the Creative Commons Attribution License, which permits unrestricted use, distribution, and reproduction in any medium, provided the original work is properly cited.

Relativistic radiative transfer in a relativistic spherical flow is examined in the fully special relativistic treatment. Under the assumption of a constant flow speed and using a variable (prescribed) Eddington factor, we analytically solve the relativistic moment equations in the comoving frame for several restricted cases, and obtain relativistic Milne-Eddington type solutions. In contrast to the plane-parallel case where the solutions exhibit the exponential behavior on the optical depth, the solutions have power-law forms. In the case of the radiative equilibrium, for example, the radiative flux has a power-law term multiplied by the exponential term. In the case of the local thermodynamic equilibrium with a uniform source function in the comoving frame, the radiative flux has a power-law form on the optical depth. This is because there is an expansion effect (curvature effect) in the spherical wind and the background density decreases as the radius increases.

\section{Introduction}

The research field of radiative transfer has been developed in astrophysics and atmospheric science [1-13]. Relativistic radiative transfer and relativistic radiation hydrodynamics have been also developed in astrophysics and applied to various energetic phenomena in the universe: nova outbursts, gamma-ray bursts, astrophysical jets, black-hole accretion disks, and black-hole winds. In the subrelativistic regime, some researchers adopted the diffusion approximation in the comoving frame (e.g., [14-17]), or proposed the variable Eddington factor (e.g., [18-24]), or performed the numerical simulations using, for example, the flux-limited diffusion (FLD) approximation (e.g., [25-35]).

In the highly relativistic regime, however, we cannot treat the relativistic radiative transfer properly. Hence, the research and development of the radiative transfer problem in relativistically moving media is now of great importance in this field.

At the present stage, the numerical approach using the FLD approximation is limited within the subrelativistic regime. In addition, even in the subrelativistic regime, the FLD method cannot reproduce the radiative force precisely in the optically thin region [36]. This is because in the optically thin region the radiative flux vector is not generally parallel to the gradient of the radiation energy density due to the effect of the distant radiation source. On the other hand, the analytical approach is very restricted in the special cases. Indeed, even for the nonrelativistic case only a few analytical solutions have been found, but for the relativistic case little is known. Recently, the relativistic radiative transfer in relativistically moving atmospheres have been investigated from the analytical view points in the plane-parallel case (e.g., [37-40]) and in the spherical case (e.g., [41]).

In Fukue [40], under the assumption of a constant flow speed, the relativistic moment equations in the comoving frame were analytically solved using a variable Eddington factor for several cases, such as the radiative equilibrium (RE) or the local thermodynamic equilibrium (LTE), and the relativistic Milne-Eddington type solutions for the relativistic plane-parallel flows have been newly found. In the present study, we also consider the relativistic radiative transfer from the analytical view point. In contrast to Fukue [40], we examine the relativistic moment equations for the spherical case, solve the equations under the assumption of a constant flow speed, and obtain several new analytical solutions for the relativistic spherical flows. 
In the next section, we describe the radiative moment equations in the comoving frame for spherical flows. In Sections 3 and 4, we show and discuss analytical solutions in the RE and LTE cases, respectively. The final section is devoted to concluding remarks.

\section{Relativistic Radiative Transfer Equation}

The radiative transfer equations are given in several standard references [6-10, 12, 13, 42-44]. The basic equations for relativistic radiation hydrodynamics are given in, for example, the Appendix E of Kato et al. [43] in general and vertical forms (see also $[39,40]$ ).

2.1. General Form. In a general form, the radiative transfer equation in the mixed frame, where the variables in the inertial and comoving frames are used, is expressed as

$$
\frac{1}{c} \frac{\partial I}{\partial t}+(\mathbf{l} \cdot \nabla) I=\left(\frac{\nu}{\nu_{0}}\right)^{3} \rho\left[\frac{j_{0}}{4 \pi}-\left(\kappa_{0}+\sigma_{0}\right) I_{0}+\sigma_{0} \frac{c E_{0}}{4 \pi}\right],
$$

where $c$ is the speed of light. In the left-hand side, the frequency-integrated specific intensity $I$ and the direction cosine vector $\mathbf{l}$ are quantities measured in the inertial (fixed) frame. In the right-hand side, on the other hand, the mass density $\rho$, the frequency-integrated mass emissivity $j_{0}$, the frequency-integrated mass absorption coefficient $\kappa_{0}$, the frequency-integrated mass scattering coefficient $\sigma_{0}$, the frequency-integrated specific intensity $I_{0}$, and the frequencyintegrated radiation energy density $E_{0}$ are quantities measured in the comoving (fluid) frame. In this paper, instead of the weakly anisotropic Thomson scattering, we assume that the scattering is isotropic for simplicity.

The Doppler effect, the aberration, and the transformation of the intensities are expressed as

$$
\begin{gathered}
\frac{\nu}{\nu_{0}}=\gamma\left(1+\boldsymbol{\beta} \cdot \mathbf{l}_{0}\right), \\
\mathbf{l}=\frac{\nu_{0}}{\nu}\left[\mathbf{l}_{0}+\left(\frac{\gamma-1}{\beta^{2}} \boldsymbol{\beta} \cdot \mathbf{1}_{0}+\gamma\right) \boldsymbol{\beta}\right], \\
I=\left(\frac{\nu}{\nu_{0}}\right)^{4} I_{0},
\end{gathered}
$$

where $v$ and $v_{0}$ are the frequencies measured in the inertial and comoving frames, respectively, the direction cosine $\mathbf{l}_{0}$ measured in the comoving frame, $\beta(=\mathrm{v} / c)$ the normalized velocity, $\mathbf{v}$ being the flow velocity, and $\gamma\left(=1 / \sqrt{1-\beta^{2}}\right)$ the Lorentz factor, $\beta$ being $\mathbf{v} / c$.

The zeroth and first moment equations are, respectively,

$$
\begin{aligned}
\frac{\partial E}{\partial t}+\frac{\partial F^{k}}{\partial x^{k}}= & \rho \gamma\left(j_{0}-\kappa_{0} c E_{0}\right)-\rho \gamma\left(\kappa_{0}+\sigma_{0}\right) \boldsymbol{\beta} \cdot \mathbf{F}_{0} \\
\frac{1}{c^{2}} \frac{\partial F^{i}}{\partial t}+\frac{\partial P^{i k}}{\partial x^{k}}= & \rho \gamma \frac{\beta^{i}}{c}\left(j_{0}-\kappa_{0} c E_{0}\right)-\rho\left(\kappa_{0}+\sigma_{0}\right) \frac{\gamma-1}{\beta^{2}} \frac{\beta^{i}}{c} \\
& \times\left(\boldsymbol{\beta} \cdot \mathbf{F}_{0}\right)-\frac{1}{c} \rho\left(\kappa_{0}+\sigma_{0}\right) F_{0}^{i}
\end{aligned}
$$

where the frequency-integrated radiation energy density $E$, the frequency-integrated radiative flux $\mathbf{F}$, and the frequencyintegrated radiation stress $P^{i k}$ are measured in the inertial frame, while those with the subscript 0 are measured in the comoving frame.

As a closure relation, we adopt the Eddington approximation in the comoving frame,

$$
P_{0}^{i k}=f^{i k} E_{0}
$$

where $f^{i k}$ is the Eddington tensor, which is generally a function of the optical depth and flow speed in the relativistic radiative flow.

2.2. Spherical Expression in the Comoving Frame. Let us suppose a relativistic spherical flow, for example, a luminous black-hole wind. In the spherical geometry with the radius $r$, the transfer equation ( 1 ) is expressed as

$$
\begin{aligned}
\frac{1}{c} \frac{\partial I}{\partial t} & +\mu \frac{\partial I}{\partial r}+\frac{1-\mu^{2}}{r} \frac{\partial I}{\partial \mu} \\
& =\left(\frac{\nu}{\nu_{0}}\right)^{3} \rho\left[\frac{j_{0}}{4 \pi}-\left(\kappa_{0}+\sigma_{0}\right) I_{0}+\sigma_{0} \frac{c E_{0}}{4 \pi}\right]
\end{aligned}
$$

where $\mu$ is the direction cosine in the inertial frame. Inserting the transformation (4) in the left-hand side, this equation (7) becomes

$$
\begin{aligned}
\frac{v}{v_{0}}\left(\frac{1}{c} \frac{\partial I_{0}}{\partial t}\right. & \left.+\mu \frac{\partial I_{0}}{\partial r}+\frac{1-\mu^{2}}{r} \frac{\partial I_{0}}{\partial \mu}\right) \\
& -4 \frac{v}{v_{0}^{2}} I_{0}\left(\frac{1}{c} \frac{\partial v_{0}}{\partial t}+\mu \frac{\partial v_{0}}{\partial z}+\frac{1-\mu^{2}}{r} \frac{\partial v_{0}}{\partial \mu}\right) \\
= & \rho\left[\frac{j_{0}}{4 \pi}-\left(\kappa_{0}+\sigma_{0}\right) I_{0}+\sigma_{0} \frac{c E_{0}}{4 \pi}\right] .
\end{aligned}
$$

To calculate the derivatives of $I_{0}$ [9], we apply the chain rules, and, after some manipulations, we have

$$
\begin{aligned}
\left.\frac{\partial}{\partial t}\right|_{r \mu \nu} & =\left.\frac{\partial}{\partial t}\right|_{r \mu_{0} \nu_{0}}+\left.\frac{\partial \mu_{0}}{\partial t}\right|_{r \mu_{0} \nu_{0}} \frac{\partial}{\partial \mu_{0}}+\left.\frac{\partial \nu_{0}}{\partial t}\right|_{r \mu_{0} \nu_{0}} \frac{\partial}{\partial \nu_{0}} \\
& =\left.\frac{\partial}{\partial t}\right|_{r \mu_{0} \nu_{0}}-\gamma^{2}\left(1-\mu_{0}^{2}\right) \frac{\partial \beta}{\partial t} \frac{\partial}{\partial \mu_{0}}-\gamma^{2} \mu_{0} \nu_{0} \frac{\partial \beta}{\partial t} \frac{\partial}{\partial \nu_{0}} \\
\left.\frac{\partial}{\partial r}\right|_{t \mu \nu} & =\left.\frac{\partial}{\partial r}\right|_{t \mu_{0} \nu_{0}}+\left.\frac{\partial \mu_{0}}{\partial r}\right|_{t \mu_{0} \nu_{0}} \frac{\partial}{\partial \mu_{0}}+\left.\frac{\partial \nu_{0}}{\partial r}\right|_{t \mu_{0} \nu_{0}} \frac{\partial}{\partial \nu_{0}} \\
& =\left.\frac{\partial}{\partial r}\right|_{t \mu_{0} \nu_{0}}-\gamma^{2}\left(1-\mu_{0}^{2}\right) \frac{\partial \beta}{\partial r} \frac{\partial}{\partial \mu_{0}}-\gamma^{2} \mu_{0} \nu_{0} \frac{\partial \beta}{\partial r} \frac{\partial}{\partial \nu_{0}} \\
\left.\frac{\partial}{\partial \mu}\right|_{r t \nu} & \left.\frac{\partial \mu_{0}}{\partial \mu}\right|_{r t v_{0}} \frac{\partial}{\partial \mu_{0}}+\left.\frac{\partial \nu_{0}}{\partial \mu_{0}}\right|_{r t \nu_{0}} \frac{\partial}{\partial \nu_{0}} \\
& =\gamma^{2}\left(1+\beta \mu_{0}\right)^{2} \frac{\partial}{\partial \mu_{0}}-\gamma^{2} \beta\left(1+\beta \mu_{0}\right) \nu_{0} \frac{\partial}{\partial \nu_{0}}
\end{aligned}
$$


where $\mu$ is the direction cosine in the comoving frame. In addition, the Doppler shift (2) and the aberration (3) are, respectively, expressed as

$$
\begin{gathered}
\frac{\nu}{\nu_{0}}=\gamma\left(1+\beta \mu_{0}\right), \\
\mu=\frac{\mu_{0}+\beta}{1+\beta \mu_{0}} .
\end{gathered}
$$

Using these expressions, after some manipulations, we have the radiative transfer equation in the comoving frame for the spherical flow:

$$
\begin{aligned}
\gamma\left(1+\beta \mu_{0}\right) & \frac{1}{c} \frac{\partial I_{0}}{\partial t}+\gamma\left(\mu_{0}+\beta\right) \frac{\partial I_{0}}{\partial r}+\gamma\left(1+\beta \mu_{0}\right) \frac{1-\mu_{0}}{r} \frac{\partial I_{0}}{\partial \mu_{0}} \\
+ & 4 \gamma \beta \frac{1-\mu_{0}^{2}}{r} I_{0}-\gamma^{3}\left(1+\beta \mu_{0}\right) \\
& \times\left[\left(1-\mu_{0}^{2}\right) \frac{\partial I_{0}}{\partial \mu_{0}}-4 \mu_{0} I_{0}\right] \frac{1}{c} \frac{\partial \beta}{\partial t} \\
& -\gamma^{3}\left(\mu_{0}+\beta\right)\left[\left(1-\mu_{0}^{2}\right) \frac{\partial I_{0}}{\partial \mu_{0}}-4 \mu_{0} I_{0}\right] \frac{\partial \beta}{\partial r} \\
= & \rho\left[\frac{j_{0}}{4 \pi}-\left(\kappa_{0}+\sigma_{0}\right) I_{0}+\sigma_{0} \frac{c E_{0}}{4 \pi}\right] .
\end{aligned}
$$

Integrating the transfer equation (11) over a solid angle, we have the zeroth and first moment equations in the comoving frame for the spherical flow:

$$
\begin{aligned}
\gamma \frac{\partial c E_{0}}{c \partial t}+ & \gamma \frac{\partial F_{0}}{\partial r}+\gamma \beta \frac{\partial F_{0}}{c \partial t}+\gamma \beta \frac{\partial c E_{0}}{\partial r}+\frac{\gamma}{r}\left[2 F_{0}+\beta\left(3 c E_{0}-c P_{0}\right)\right] \\
& +\gamma^{3}\left[2 F_{0}+\beta\left(c E_{0}+c P_{0}\right)\right] \frac{\partial \beta}{c \partial t} \\
& +\gamma^{3}\left[2 \beta F_{0}+\left(c E_{0}+c P_{0}\right)\right] \frac{\partial \beta}{\partial r} \\
= & \rho\left(j_{0}-\kappa_{0} c E_{0}\right), \\
\gamma \frac{\partial F_{0}}{c \partial t}+ & \gamma \frac{\partial c P_{0}}{\partial r}+\gamma \beta \frac{\partial c P_{0}}{c \partial t}+\gamma \beta \frac{\partial F_{0}}{\partial r}+\frac{\gamma}{r}\left[2 \beta F_{0}-c E_{0}+3 c P_{0}\right] \\
& +\gamma^{3}\left[2 \beta F_{0}+\left(c E_{0}+c P_{0}\right)\right] \frac{\partial \beta}{c \partial t} \\
& +\gamma^{3}\left[2 F_{0}+\beta\left(c E_{0}+c P_{0}\right)\right] \frac{\partial \beta}{\partial r} \\
= & -\rho\left(\kappa_{0}+\sigma_{0}\right) F_{0},
\end{aligned}
$$

where $E_{0}, F_{0}$, and $P_{0}$ are the radiation energy density, the radiative flux, and the radiation pressure in the comoving frame, respectively.

In the present spherical one-dimensional flow, if we assume the pressure isotropy in the comoving frame, the closure relation (6) becomes

$$
P_{0}=f(\tau, \beta) E_{0},
$$

where $f(\tau, \beta)$ is the variable (prescribed) Eddington factor, and generally a function of the optical depth, the flow speed, and the velocity gradient $[45,46]$. In the plane-parallel flow [40], the following form was adopted:

$$
f(\beta)=\frac{1+3 \beta^{2}}{3+\beta^{2}},
$$

which is $1 / 3$ for $\beta=0$ and approaches unity as $\beta \rightarrow 1$ [47]. In the present spherical case, we adopt alternative appropriate forms, which are shown later.

It should be noted that historically Auer and Mihalas [48] first used the term a variable Eddington factor $(V E F)$ to express an iterative solver to obtain the Eddington factor (cf. [49]). The Eddington factors which depend on the optical depth should be called prescribed or approximated Eddington factors, although they are often called a variable Eddington factor $[50,51]$. In the previous papers, in order to express the not-constant Eddington factor, we also used the variable Eddington factor, which depends on the optical depth and flow velocity. In this paper we use both terms, variable and prescribed, but both usages express the same meanings; that is, the present Eddington factor is not constant but varies as a function of the optical depth and flow velocity.

2.3. Steady Spherical Flow. Let us further suppose a timeindependent steady flow in the radial direction. In this case the transfer equation and moment equations in the comoving frame become

$$
\begin{aligned}
& \gamma\left(\mu_{0}+\beta\right) \frac{d I_{0}}{d r}-\gamma^{3}\left(\mu_{0}+\beta\right)\left[\left(1-\mu_{0}^{2}\right) \frac{\partial I_{0}}{\partial \mu_{0}}-4 \mu_{0} I_{0}\right] \frac{d \beta}{d r} \\
&+\gamma\left(1+\beta \mu_{0}\right) \frac{1-\mu_{0}^{2}}{r} \frac{\partial I_{0}}{\partial \mu_{0}}+4 \gamma \beta \frac{1-\mu_{0}^{2}}{r} I_{0} \\
&= \rho\left[\frac{j_{0}}{4 \pi}-\left(\kappa_{0}+\sigma_{0}\right) I_{0}+\sigma_{0} \frac{c E_{0}}{4 \pi}\right], \\
& \gamma \frac{d F_{0}}{d r}+ \gamma \beta \frac{d c E_{0}}{d r}+\gamma^{3}\left[2 \beta F_{0}+\left(c E_{0}+c P_{0}\right)\right] \frac{d \beta}{d r} \\
&+\frac{\gamma}{r}\left[2 F_{0}+\beta\left(3 c E_{0}-c P_{0}\right)\right]=\rho\left(j_{0}-\kappa_{0} c E_{0}\right), \\
& \gamma \frac{d c P_{0}}{d r}+\gamma \beta \frac{d F_{0}}{d r}+\gamma^{3}\left[2 F_{0}+\beta\left(c E_{0}+c P_{0}\right)\right] \frac{d \beta}{d r} \\
&+\frac{\gamma}{r}\left[2 \beta F_{0}-c E_{0}+3 c P_{0}\right]=-\rho\left(\kappa_{0}+\sigma_{0}\right) F_{0} .
\end{aligned}
$$

Introducing the optical depth defined by

$$
d \tau \equiv-\left(\kappa_{0}+\sigma_{0}\right) \rho d r
$$

and the scattering albedo,

$$
a \equiv \frac{\sigma_{0}}{\kappa_{0}+\sigma_{0}},
$$


the transfer equation (15) and the moment equations (16) and (17) are finally expressed as

$$
\begin{aligned}
\gamma\left(\mu_{0}+\beta\right) & \frac{d I_{0}}{d \tau}-\gamma\left(1+\beta \mu_{0}\right) \frac{1-\mu_{0}^{2}}{\rho\left(\kappa_{0}+\sigma_{0}\right) r} \frac{\partial I_{0}}{\partial \mu_{0}} \\
& -4 \gamma \beta \frac{1-\mu_{0}^{2}}{\rho\left(\kappa_{0}+\sigma_{0}\right) r} I_{0} \\
& -\gamma^{3}\left(\mu_{0}+\beta\right)\left[\left(1-\mu_{0}^{2}\right) \frac{\partial I_{0}}{\partial \mu_{0}}-4 \mu_{0} I_{0}\right] \frac{d \beta}{d \tau} \\
= & I_{0}-\frac{1}{4 \pi} \frac{j_{0}}{\kappa_{0}+\sigma_{0}}-a \frac{c E_{0}}{4 \pi}, \\
\gamma \frac{d F_{0}}{d \tau}+ & \gamma \beta \frac{d c E_{0}}{d \tau}-\frac{\gamma}{\rho\left(\kappa_{0}+\sigma_{0}\right) r}\left[2 F_{0}+\beta\left(3 c E_{0}-c P_{0}\right)\right] \\
& +\gamma^{3}\left[2 \beta F_{0}+\left(c E_{0}+c P_{0}\right)\right] \frac{d \beta}{d \tau} \\
= & -\frac{j_{0}}{\kappa_{0}+\sigma_{0}}+(1-a) c E_{0}, \\
= & F_{0} . \\
\gamma \frac{d c P_{0}}{d \tau}+ & \gamma \beta \frac{d F_{0}}{d \tau}-\frac{\gamma}{\rho\left(\kappa_{0}+\sigma_{0}\right) r}\left[2 \beta F_{0}-c E_{0}+3 c P_{0}\right] \\
& +\gamma^{3}\left[2 F_{0}+\beta\left(c E_{0}+c P_{0}\right)\right] \frac{d \beta}{d \tau}
\end{aligned}
$$

Here, we further introduce the spherical variables by

$$
\begin{aligned}
L_{0} & \equiv 4 \pi r^{2} F_{0}, \\
D_{0} & \equiv 4 \pi r^{2} c E_{0}, \\
Q_{0} & \equiv 4 \pi r^{2} c P_{0},
\end{aligned}
$$

and the moment equations (21) and (22) become

$$
\begin{aligned}
\gamma \frac{d L_{0}}{d \tau} & +\gamma \beta \frac{d D_{0}}{d \tau}-\gamma \beta \frac{D_{0}-Q_{0}}{\rho\left(\kappa_{0}+\sigma_{0}\right) r}+\gamma^{3}\left(2 \beta L_{0}+D_{0}+Q_{0}\right) \frac{d \beta}{d \tau} \\
= & -\frac{4 \pi r^{2}}{\kappa_{0}+\sigma_{0}}\left(j_{0}-\kappa_{0} c E_{0}\right), \\
\gamma \frac{d Q_{0}}{d \tau} & +\gamma \beta \frac{d L_{0}}{d \tau}-\gamma \frac{Q_{0}-D_{0}}{\rho\left(\kappa_{0}+\sigma_{0}\right) r}+\gamma^{3}\left[2 L_{0}+\beta\left(D_{0}+Q_{0}\right)\right] \frac{d \beta}{d \tau} \\
= & L_{0},
\end{aligned}
$$

and the closure relation (13) is written as

$$
Q_{0}=f(\tau, \beta) D_{0}
$$

If we assume the streaming limit of $D_{0}=Q_{0}(f=1)$ with a constant speed in (24), we have the exponential type solutions. In this paper we consider more general cases of $Q_{0}=f D_{0}$.
Using this closure relation (25), the Eddington factor being not yet determined, and the definition of the optical depth (18), the relativistic moment equation (24) is expressed as

$$
\begin{aligned}
& \gamma \frac{d L_{0}}{d \tau}+\gamma \beta \frac{d D_{0}}{d \tau}+\gamma \beta \frac{1-f}{r} D_{0} \frac{d r}{d \tau}+\gamma^{3}\left[2 \beta L_{0}+(1+f) D_{0}\right] \frac{d \beta}{d \tau} \\
& =-\frac{4 \pi r^{2}}{\kappa_{0}+\sigma_{0}}\left(j_{0}-\kappa_{0} c E_{0}\right), \\
& \gamma \frac{d\left(f D_{0}\right)}{d \tau}+\gamma \beta \frac{d L_{0}}{d \tau}-\gamma \frac{1-f}{r} D_{0} \frac{d r}{d \tau} \\
& +\gamma^{3}\left[2 L_{0}+\beta(1+f) D_{0}\right] \frac{d \beta}{d \tau} \\
& =L_{0} .
\end{aligned}
$$

After several manipulations and rearrangement, the relativistic moment equation (26) in the comoving frame is finally expressed as

$$
\begin{aligned}
& \frac{\gamma\left(f-\beta^{2}\right)}{f} \frac{d L_{0}}{d \tau}+\gamma \beta\left(\frac{1-f^{2}}{f r}-\frac{1}{f} \frac{d f}{d r}\right) D_{0} \frac{d r}{d \tau} \\
& +\gamma^{3}\left[2 \beta\left(1-\frac{1}{f}\right) L_{0}+(1+f)\left(1-\frac{\beta^{2}}{f}\right) D_{0}\right] \frac{d \beta}{d \tau} \\
& =-\frac{4 \pi r^{2}}{\kappa_{0}+\sigma_{0}}\left(j_{0}-\kappa_{0} c E_{0}\right)-\frac{\beta}{f} L_{0}, \\
& \gamma\left(f-\beta^{2}\right) \frac{d D_{0}}{d \tau}+\gamma\left[\frac{d f}{d r}-\frac{(1-f)\left(1+\beta^{2}\right)}{r}\right] D_{0} \frac{d r}{d \tau} \\
& +2 \gamma L_{0} \frac{d \beta}{d \tau} \\
& =L_{0}+\beta \frac{4 \pi r^{2}}{\kappa_{0}+\sigma_{0}}\left(j_{0}-\kappa_{0} c E_{0}\right) .
\end{aligned}
$$

After we determine the appropriate form of the variable Eddington factor, we can solve the moment equation (27) in some restricted cases.

Before solving the moment equations, we derive a relation between the optical depth $\tau$ and radius $r$. If the flow is steady, as is assumed, the continuity equation for the spherical case is written as

$$
4 \pi r^{2} \rho \gamma \beta c=\dot{M}
$$

where $\dot{M}$ is the constant mass-outflow rate. Using this continuity equation (28), assuming the opacities are constant, and imposing the boundary condition of $\tau=0$ at $r=\infty$, we can integrate the optical depth (18) to give

$$
\tau=\frac{\dot{M}\left(\kappa_{0}+\sigma_{0}\right)}{4 \pi \gamma \beta c} \frac{1}{r}=\rho\left(\kappa_{0}+\sigma_{0}\right) r,
$$

which is also written as

$$
\frac{\tau}{\tau_{\mathrm{c}}}=\frac{r_{\mathrm{c}}}{r}
$$


where the subscript $c$ denotes some reference position (core radius). It should be noted that the optical depth at the core radius is related to the core radius by

$$
\tau_{\mathrm{c}}=\frac{\dot{m} r_{\mathrm{g}}}{2 \gamma \beta r_{\mathrm{c}}}
$$

where $\dot{m}\left(=\dot{M} / \dot{M}_{\mathrm{E}}\right)$ is the mass-outflow rate normalized by the critical rate $\dot{M}_{\mathrm{E}}\left(=L_{\mathrm{E}} / c^{2}\right), L_{\mathrm{E}}$ being the Eddington luminosity of the central object, and $r_{\mathrm{g}}\left(=2 \mathrm{GM} / \mathrm{c}^{2}\right)$ is the Schwarzshild radius of the central object. In what follows, we use these relations, if necessary.

\section{Radiative Equilibrium}

We first consider the case of the radiative equilibrium (RE) without heating and cooling. If the radiative equilibrium holds in the whole flow, and there is no heating or cooling, then $j_{0}=\kappa_{0} c E_{0}$, and the relativistic moment equation (27) become

$$
\begin{aligned}
& \frac{\gamma\left(f-\beta^{2}\right)}{f} \frac{d L_{0}}{d \tau}+\gamma \beta\left(\frac{1-f^{2}}{f r}-\frac{1}{f} \frac{d f}{d r}\right) D_{0} \frac{d r}{d \tau} \\
& \quad+\gamma^{3}\left[2 \beta\left(1-\frac{1}{f}\right) L_{0}+(1+f)\left(1-\frac{\beta^{2}}{f}\right) D_{0}\right] \frac{d \beta}{d \tau} \\
& =-\frac{\beta}{f} L_{0} \\
& \gamma\left(f-\beta^{2}\right) \frac{d D_{0}}{d \tau}+\gamma\left[\frac{d f}{d r}-\frac{(1-f)\left(1+\beta^{2}\right)}{r}\right] D_{0} \frac{d r}{d \tau} \\
& \quad+2 \gamma L_{0} \frac{d \beta}{d \tau} \\
& =L_{0} .
\end{aligned}
$$

These equations (32) and (33) are rather complicated yet, since they include the velocity gradient term and the derivative of the radius, which are connected with the hydrodynamical equations. Of these, except for the central accelerating region, the wind speed weakly depends on the optical depth and is almost constant in the terminal stage. Hence, as already stated, the flow speed $\beta$ is assumed to be constant in this paper. On the other hand, the radiusderivative term depends on the optical depth. Indeed, it is expressed as

$$
\frac{d r}{d \tau}=-\frac{1}{\left(\kappa_{0}+\sigma_{0}\right) \rho}=-\frac{r}{\tau} \propto-\tau^{-2} .
$$

Instead, the second term on the left-hand side of (33) can be dropped, if we impose the restricted condition on the Eddington factor as

$$
\frac{1-f^{2}}{r}-\frac{d f}{d r}=0
$$

This equation (35) is easily integrated to give

$$
f=\frac{C(\beta) r^{2}-1}{C(\beta) r^{2}+1}
$$

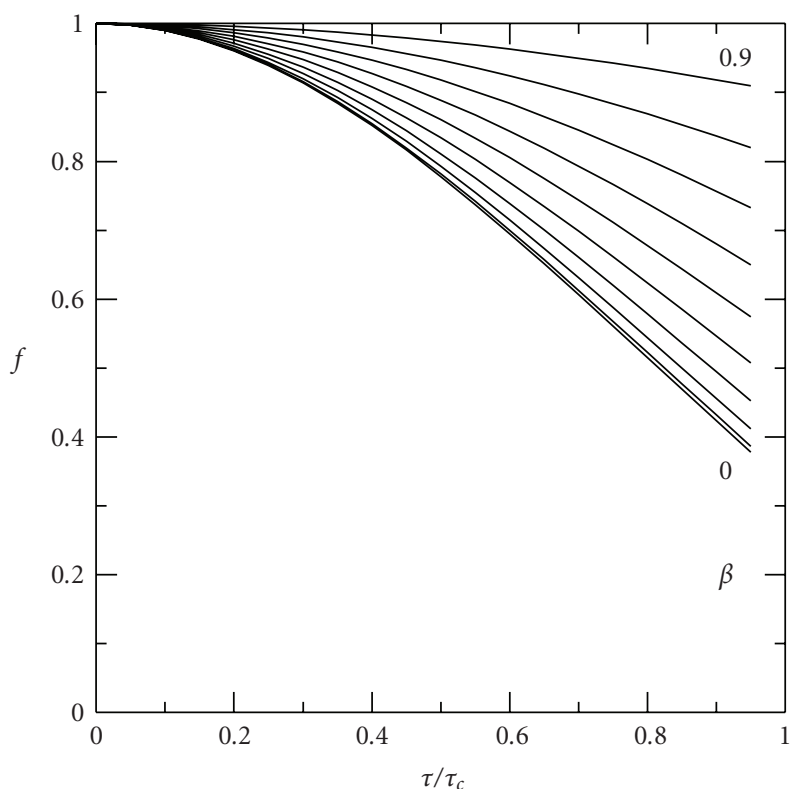

FIGURE 1: Eddington factor $f$ appropriate for the RE case as a function of the optical depth $\tau$ for the various values of the flow speed $\beta$. The values of $\beta$ are 0 to 0.9 in steps of from 0.1 from bottom to top.

where $C(\beta)$ is an integration constant, and generally a function of the constant flow speed $\beta$. We impose the boundary condition at the core radius such as

$$
f=\frac{1+3 \beta^{2}}{3+\beta^{2}} \text { at } r=r_{\mathrm{c}},
$$

and the appropriate Eddington factor requested to the present case finally becomes

$$
f=\frac{2 \gamma^{2}\left(1+\beta^{2}\right) \hat{r}^{2}-1}{2 \gamma^{2}\left(1+\beta^{2}\right) \hat{r}^{2}+1}=\frac{2 \gamma^{2}\left(1+\beta^{2}\right)-\hat{\tau}^{2}}{2 \gamma^{2}\left(1+\beta^{2}\right)+\hat{\tau}^{2}},
$$

where $\hat{r}=r / r_{\mathrm{c}}$ and $\hat{\tau}=\tau / \tau_{\mathrm{c}}$. This variable Eddington factor (38) satisfies the condition $f \rightarrow 1 / 3$ when $r \rightarrow r_{\mathrm{c}}$ and $\beta \rightarrow$ 0 , and $f \rightarrow 1$ when $r \rightarrow \infty(\tau \rightarrow 0)$ or $\beta \rightarrow 1$. The behavior of this Eddington factor is shown in Figure 1.

Under these restrictive conditions, after several manipulations, (32) and (33) become

$$
\begin{gathered}
\frac{d L_{0}}{d \tau}=-\Gamma L_{0}, \\
\gamma \frac{1}{g} \frac{d}{d \tau}\left[g\left(f-\beta^{2}\right) D_{0}\right]=L_{0} .
\end{gathered}
$$

In these equations,

$$
\Gamma \equiv \frac{\beta}{\gamma\left(f-\beta^{2}\right)}
$$

is a function of the flow speed and the optical depth, and it becomes

$$
\Gamma=\frac{\gamma \beta}{1+\beta^{2}} \frac{2 \hat{\tau}^{2}}{2-\hat{\tau}^{2}}+\gamma \beta
$$




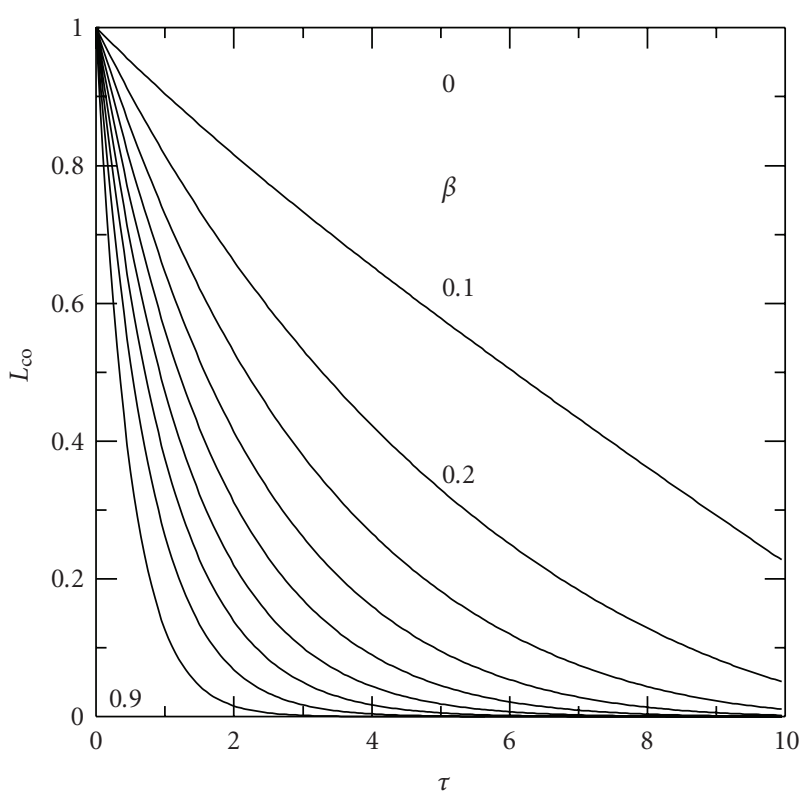

Figure 2: Comoving luminosity for relativistic spherical flows in the RE case without heating and cooling. The values of $\beta$ are from 0 to 0.9 in steps of 0.1 from top to bottom. The optical depth $\tau_{\mathrm{c}}$ at the core radius is set to be 10 .

for the Eddington factor (38), while $g$ is the curvature factor defined by

$$
\ln g \equiv-\int_{\tau_{c}}^{\tau} \frac{(1-f)\left(1+\beta^{2}\right)}{\left(f-\beta^{2}\right) r} \frac{d r}{d \tau} d \tau^{\prime}
$$

and becomes in the present case

$$
g=\frac{\hat{\tau}^{3}}{2-\hat{\tau}^{2}} .
$$

Since the index $\Gamma$ is analyticalls expressed by the optical depth, the differential equation (39) can analytically integrate to give the comoving luminosity $L_{0}$. Imposing the boundary condition of $L_{\mathrm{s}}$ at $\tau=0$, we finally have the comoving luminosity for the RE case:

$$
\frac{L_{0}}{L_{\mathrm{s}}}=\left(\frac{\sqrt{2}-\hat{\tau}}{\sqrt{2}+\hat{\tau}}\right)^{b} \exp \left(\frac{1}{\sqrt{2} \gamma^{2}} b \hat{\tau}\right)
$$

where

$$
b=\frac{\sqrt{2} \gamma \beta}{1+\beta^{2}} \tau_{\mathrm{c}} .
$$

The analytical solutions of the comoving luminosity (45) are shown in Figure 2 as a function of the optical depth for several values of the flow speed. The values of $\beta$ are from 0 to 0.9 in steps of 0.1 .

Although the comoving luminosity (45) has an exponential term, the power-law behavior is dominant in this case. In the nonrelativistic limit of $\beta \rightarrow 0, b \rightarrow 0$ and the solution reduces to

$$
\frac{L_{0}}{L_{\mathrm{s}}} \sim 1-\frac{b}{\sqrt{2}} \hat{\tau} \sim 1-\beta \tau
$$

In the extremely relativistic limit of $\beta \rightarrow 1$, on the other hand, $b \rightarrow \gamma \tau_{c} / \sqrt{2}$ and the solution reduces to

$$
\frac{L_{0}}{L_{\mathrm{s}}} \sim\left(\frac{\sqrt{2}-\hat{\tau}}{\sqrt{2}+\hat{\tau}}\right)^{b}
$$

In contrast to this comoving luminosity, it is still difficult to obtain analytical solutions of the spherical radiation energy density $D_{0}$. Even in the extremely relativistic limit, we cannot obtain the analytical solution for $D_{0}$.

\section{Local Thermodynamic Equilibrium}

Next, we consider the case of the local thermodynamic equilibrium (LTE) with a uniform source function. If the local thermodynamic equilibrium (LTE) holds in the comoving frame,

$$
\frac{j_{0}}{4 \pi}=\kappa_{0} B_{0}
$$

where $B_{0}\left(=\sigma T_{0}^{4} / \pi\right)$ is the frequency-integrated blackbody intensity in the comoving frame, $T_{0}$ being the blackbody temperature and generally a function of the height $r$ or the optical depth $\tau$, but assumed to be constant in what follows.

In this case the relativistic moment equation (27) become

$$
\begin{aligned}
& \frac{\gamma\left(f-\beta^{2}\right)}{f} \frac{d L_{0}}{d \tau}+\gamma \beta\left(\frac{1-f^{2}}{f r}-\frac{1}{f} \frac{d f}{d r}\right) D_{0} \frac{d r}{d \tau} \\
& +\gamma^{3}\left[2 \beta\left(1-\frac{1}{f}\right) L_{0}+(1+f)\left(1-\frac{\beta^{2}}{f}\right) D_{0}\right] \frac{d \beta}{d \tau} \\
& =-\frac{\kappa_{0}}{\kappa_{0}+\sigma_{0}}\left(W_{0}-D_{0}\right)-\frac{\beta}{f} L_{0}, \\
& \gamma\left(f-\beta^{2}\right) \frac{d D_{0}}{d \tau}+\gamma\left[\frac{d f}{d r}-\frac{(1-f)\left(1+\beta^{2}\right)}{r}\right] \\
& \quad \times D_{0} \frac{d r}{d \tau}+2 \gamma L_{0} \frac{d \beta}{d \tau} \\
& =L_{0}+\beta \frac{\kappa_{0}}{\kappa_{0}+\sigma_{0}}\left(W_{0}-D_{0}\right),
\end{aligned}
$$

where

$$
W_{0} \equiv 16 \pi^{2} r^{2} B_{0}
$$

is the spherical source function. 


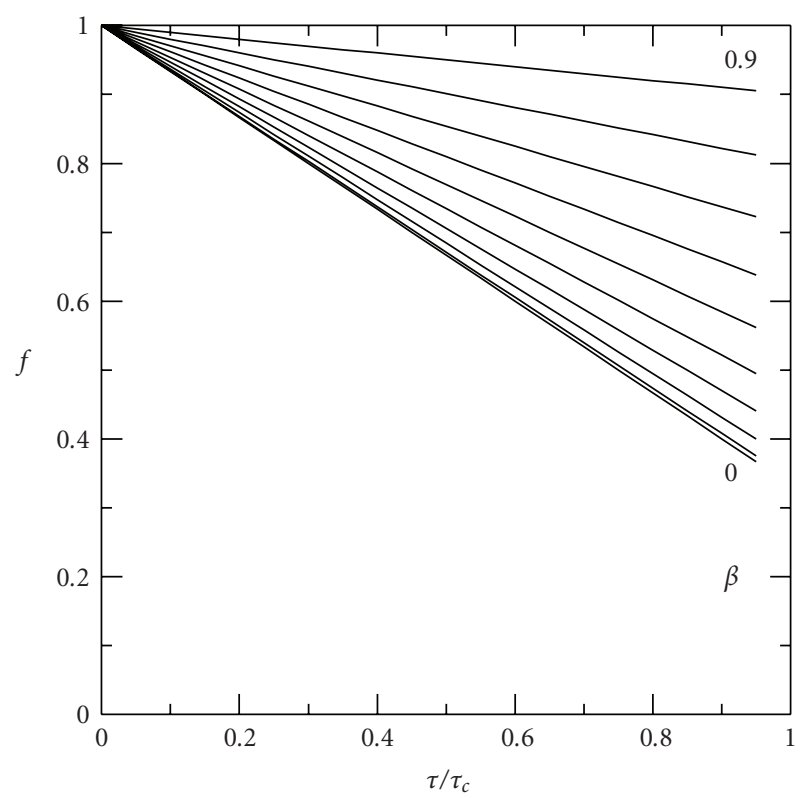

FIGURE 3: Eddington factor $f$ appropriate for the LTE case as a function of the optical depth $\tau$ for the various values of the flow speed $\beta$. The values of $\beta$ are from 0 to 0.9 in steps of 0.1 from bottom to top.

These equations (50) and (51) can be rearranged as

$$
\begin{aligned}
& \frac{\gamma\left(f-\beta^{2}\right)}{f} \frac{d L_{0}}{d \tau}+\left[\frac{\gamma \beta}{f}\left(\frac{1-f^{2}}{r}-\frac{d f}{d r}\right) \frac{d r}{d \tau}-\frac{\kappa_{0}}{\kappa_{0}+\sigma_{0}}\right] D_{0} \\
&+ \gamma^{3}\left[2 \beta\left(1-\frac{1}{f}\right) L_{0}+(1+f)\left(1-\frac{\beta^{2}}{f}\right) D_{0}\right] \frac{d \beta}{d \tau} \\
&=-\frac{\kappa_{0}}{\kappa_{0}+\sigma_{0}} W_{0}-\frac{\beta}{f} L_{0}, \\
& \gamma\left(f-\beta^{2}\right) \frac{d D_{0}}{d \tau} \\
&+\left\{\gamma\left[\frac{d f}{d r}-\frac{(1-f)\left(1+\beta^{2}\right)}{r}\right] \frac{d r}{d \tau}+\beta \frac{\kappa_{0}}{\kappa_{0}+\sigma_{0}}\right\} \\
& \quad \times D_{0}+2 \gamma L_{0} \frac{d \beta}{d \tau} \\
&=L_{0}+\beta \frac{\kappa_{0}}{\kappa_{0}+\sigma_{0}} W_{0} .
\end{aligned}
$$

Equation (52) is yet too complicated to solve analytically. Hence, in order to simplify these equations by dropping the second terms on the left-hand sides of equation (52), we impose the following two conditions:

$$
\begin{gathered}
\frac{\gamma \beta}{f}\left(\frac{1-f^{2}}{r}-\frac{d f}{d r}\right) \frac{d r}{d \tau}-\frac{\kappa_{0}}{\kappa_{0}+\sigma_{0}}=0, \\
\gamma\left[\frac{d f}{d r}-\frac{(1-f)\left(1+\beta^{2}\right)}{r}\right] \frac{d r}{d \tau}+\beta \frac{\kappa_{0}}{\kappa_{0}+\sigma_{0}}=0 .
\end{gathered}
$$

Eliminating $\kappa_{0} /\left(\kappa_{0}+\sigma_{0}\right)$ from (53), we obtain the differential equation for the variable Eddington factor $f$,

$$
\frac{d f}{d r}+\frac{f-1}{r}=0
$$

as long as $d r / d \tau \neq 0$.

This equation (54) is easily integrated to give

$$
f=1-\frac{C(\beta)}{\hat{r}},
$$

where $C(\beta)$ is an integration constant and generally a function of the constant flow speed $\beta$. We impose the boundary condition at the core radius such as

$$
f=\frac{1+3 \beta^{2}}{3+\beta^{2}} \text { at } r=r_{\mathrm{c}}
$$

and the appropriate Eddington factor requested to the present case finally becomes

$$
f=1-\frac{2\left(1-\beta^{2}\right)}{3+\beta^{2}} \frac{1}{\hat{r}}=1-\frac{2\left(1-\beta^{2}\right)}{3+\beta^{2}} \hat{\tau},
$$

where $\hat{r}=r / r_{\mathrm{c}}$ and $\hat{\tau}=\tau / \tau_{\mathrm{c}}$. This variable Eddington factor (57) satisfies the condition: $f \rightarrow 1 / 3$ when $r \rightarrow r_{\mathrm{c}}$ and $\beta \rightarrow$ 0 , and $f \rightarrow 1$ when $r \rightarrow \infty(\tau \rightarrow 0)$ or $\beta \rightarrow 1$. The behavior of this Eddington factor is shown in Figure 3.

Under these restrictive conditions, after several manipulations, equation (52) becomes

$$
\begin{aligned}
& \frac{d L_{0}}{d \tau}=-\Gamma L_{0}-\Delta \frac{\kappa_{0}}{\kappa_{0}+\sigma_{0}} W_{0}, \\
& \frac{d D_{0}}{d \tau}=\frac{\Gamma}{\beta} L_{0}+\Gamma \frac{\kappa_{0}}{\kappa_{0}+\sigma_{0}} W_{0},
\end{aligned}
$$

where

$$
\begin{aligned}
& \Gamma \equiv \frac{\beta}{\gamma\left(f-\beta^{2}\right)}=\frac{\gamma \beta\left(3+\beta^{2}\right)}{3+\beta^{2}-2 \hat{\tau}}, \\
& \Delta \equiv \frac{f}{\gamma\left(f-\beta^{2}\right)}=\gamma \frac{3+\beta^{2}-2\left(1-\beta^{2}\right) \hat{\tau}}{3+\beta^{2}-2 \hat{\tau}},
\end{aligned}
$$

respectively, in the present case.

Since the index $\Gamma$ is analytically expressed by the optical depth, the solution of the homegeneous part of (58), where $W_{0}$ is set to be 0 , is analytically obtained as

$$
\frac{L_{0}}{L_{\mathrm{s}}}=(1-p \hat{\tau})^{q},
$$

where

$$
\begin{gathered}
p \equiv \frac{2}{3+\beta^{2}}, \\
q \equiv \frac{\gamma \beta\left(3+\beta^{2}\right)}{2} \tau_{c} .
\end{gathered}
$$




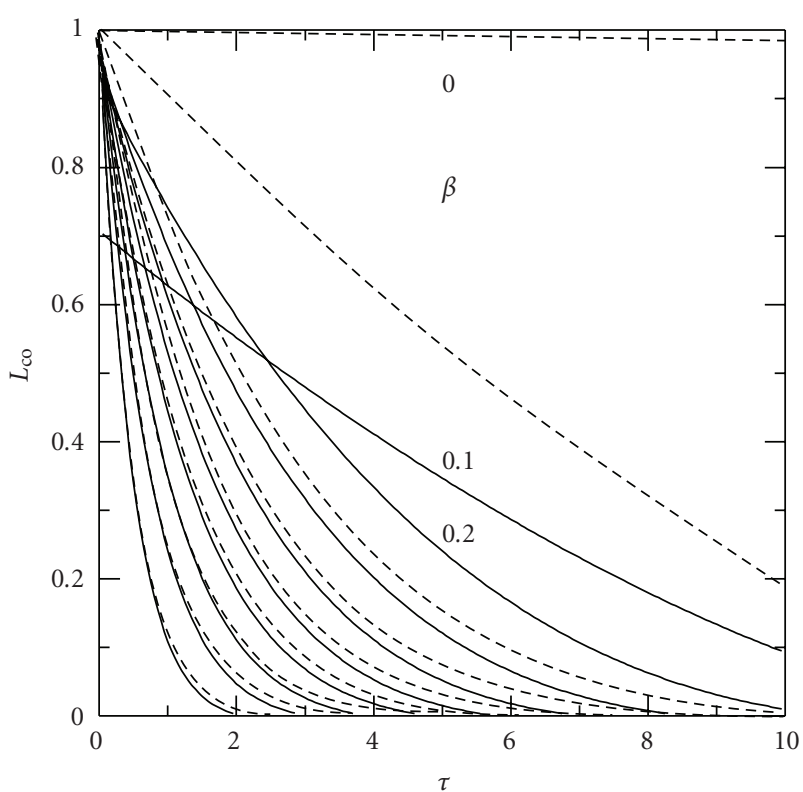

Figure 4: Comoving luminosity for relativistic spherical flows in the LTE case with a uniform source function. The values of $\beta$ are from 0 to 0.9 in steps of 0.1 from top to bottom. The optical depth $\tau_{\mathrm{c}}$ at the core radius is set to be 10 , and the value of the source function $\left[\kappa_{0} /\left(\kappa_{0}+\sigma_{0}\right)\right] W_{0} / L_{\mathrm{s}}$ is set to be unity. The solid curves represent the present analytical solutions, while the dashed ones mean the solutions of the homogeneous part.

When the spherical source function $W_{0}$ is uniform and $\kappa_{0} /\left(\kappa_{0}+\sigma_{0}\right)$ is also constant, the analytical solution of (58) can be obtained after some manipulations as

$$
\begin{aligned}
\frac{L_{0}}{L_{\mathrm{s}}}= & (1-p \hat{\tau})^{q} \\
& +\frac{\gamma \tau_{\mathrm{c}}}{1-q}\left[\left(\frac{3+\beta^{2}}{2}-\frac{\beta}{\gamma \tau_{\mathrm{c}}}\right)-\frac{\hat{\tau}}{\gamma^{2}}\right] \frac{\kappa_{0}}{\kappa_{0}+\sigma_{0}} \frac{W_{0}}{L_{\mathrm{s}}} .
\end{aligned}
$$

The analytical solutions of the comoving luminosity (63) are shown in Figure 4 as a function of the optical depth for several values of the flow speed. The values of $\beta$ are from 0 to 0.9 in steps of 0.1 .

In the LTE case the comoving luminosity (63) has the power-law form. In the nonrelativistic limit of $\beta \rightarrow 0, p \rightarrow$ $2 / 3$, and $q \rightarrow 3 \beta \tau_{\mathrm{c}} / 2$, and the solution becomes a linear function of $\tau$. In the extremely relativistic limit of $\beta \rightarrow 1$, on the other hand, $p \rightarrow 1 / 2$ and $q \rightarrow 2 \gamma \tau_{c}$, the solution reduces to

$$
\frac{L_{0}}{L_{\mathrm{s}}} \sim(1-p \hat{\tau})^{q}-\frac{\kappa_{0}}{\kappa_{0}+\sigma_{0}} \frac{W_{0}}{L_{\mathrm{s}}} .
$$

In contrast to the $\mathrm{RE}$ case, we can obtain the analytical solutions of the spherical radiation energy density $D_{0}$. However, it is rather complicated, and we omit the expression for $D_{0}$.

\section{Concluding Remarks}

In this paper, we have examined the relativistic radiative transfer in the relativistic spherical flows in the fully special relativistic treatment. Under the assumption of a constant flow speed and using a variable Eddington factor $f(\tau, \beta)$, we have analytically solved the relativistic moment equations written in the comoving frame for RE and LTE cases and found new analytical solutions for several restricted situations. In both RE and LTE cases, the radiative flux decreases with the optical depth in the power-law manner, while the radiative flux has the exponential behavior in the plane-parallel case [40].

We here clarify the essential difference for the relativistic radiative transfer between the plane-parallel and spherical cases; the former is the exponential type, and the latter is the power-law manner. Since the original transfer equation is the linear differential equation, the exponential behavior is natural, but there arised two different types. This essential difference is roughly understood as follows.

In the relativistic plane-parallel flow [40], where we have assumed the constant flow speed, the density is also constant; there is no expansion effect. The index $\Gamma$ is also constant. In this case, the natural exponential behavior emerges and the analytical solutions exhibit the exponential behavior on the optical depth. In the relativistic spherical flow in the present case, where we have also assumed the constant flow speed, the density decreases as the radius increases due to the geometrical effect; there is an expansion effect. The index $\Gamma$ is no longer constant but varies as a function of $r$ (or $\tau$ ). As a result, the natural exponential behavior is lost, and the analytical solutions exhibit the power-law behavior.

From the view point of the background density variation, this difference is somewhat similar to the growth of the density fluctuation of the gravitational instability in the static interstellar space and the expanding universe. Namely, in the static background, where the background density is constant, the density fluctuation increases exponentially [52], whereas it increases in a power-law manner in the expanding universe, where the background density decreases with time [53]. Hence, we can guess that, even in the planeparallel case, there may be power-law type solutions if the flow is accelerated and the density decreases as the optical depth decreases.

In order to research the physical problem, the analytical approach has several advantages. First, the analytical solutions can often reveal the essential properties of the radiative transfer problem. In the present case, we can clarify the exponential versus power-law type behavior and its causes. Secondly, they can clarify the restrictions of the assumptions and/or crucial problems inherent in the formalism. In the present case, in order to avoid the critical point in the basic equations with a traditional constant Eddington factor, we use a variable Eddington factor, which approaches unity in the limit of $\tau \rightarrow 0$ or $\beta \rightarrow 1$ (cf. [24, 54]). Finally, they can help us to check the precision and the validity of the numerical code for the radiative transfer problem. Particularly, in the recent research of the radiation transfer problem on the black-hole accretion using the ART code [36], the FLD approximation often adopted in the radiation hydrodynamical simulations cannot reproduce the radiative force in the optically thin region. Hence, the new numerical method should be developed for the multidimensional 
radiation hydrodynamical simulations, and the analytical solutions like the present case would be useful for such codes in the future.

\section{Acknowledgment}

The author would like to thank an anonymous referee for valuable comments. This work has been supported in part by the Grant-in-Aid for Scientific Research (C) of the Ministry of Education, Culture, Sports, Science and Technology (22540251 JF).

\section{References}

[1] A. Schuster, "Radiation through a foggy atmosphere," Astrophysicat Journal, vol. 21, pp. 1-22, 1905.

[2] E. A. Milne, "Radiative equilibrium in the outer layers of a star," Monthly Notices of the Royal Astronomical Society, vol. 81, pp. 361-375, 1921.

[3] A. S. Eddington, The Internal Consititution of Stars, Cambridge University Press, Cambridge, UK, 1926.

[4] N. A. Kosirev, "Radiative equilibrium of the extended photosphere," Monthly Notices of the Royal Astronomical Society, vol. 94, pp. 430-443, 1934.

[5] S. Chandrasekhar, "The radiative equilibrium of extended stellar atmospheres," Monthly Notices of the Royal Astronomical Society, vol. 94, pp. 444-458, 1934.

[6] S. Chandrasekhar, Radiative Transfer, Dover Publishing, New York, NY, USA, 1960.

[7] D. Mihalas, Stellar Atmospheres, W.H. Freeman and Co., San Francisco, Calif, USA, 1970.

[8] G. B. Rybicki and A. P. Lightman, Radiative Processes in Astrophysics, John Wiley \& Sons, New York, NY, USA, 1979.

[9] D. Mihalas and B. W. Mihalas, Foundations of Radiation Hydrodynamics, Oxford University Press, Oxford, UK, 1984.

[10] F. H. Shu, The Physics of Astrophysics Vol. 1: Radiation, University Science Books, Mill Valley, Calif, USA, 1991.

[11] G. E. Thomas and K. Stamnes, Radiative Transfer in the Atmosphere and Ocean, Cambridge University Press, Cambridge, UK, 1999.

[12] A. Peraiah, An Introduction to Radiative Transfer: Methods and Applications in Astrophysics, Cambridge University Press, Cambridge, UK, 2002.

[13] J. I. Castor, Radiation Hydrodynamics, Cambridge University Press, Cambridge, UK, 2004.

[14] C. L. N. Ruggles and G.T. Bath, "The structure of optically thick winds in classical novae," Astronomy and Astrophysics, vol. 80, no. 1, pp. 97-103, 1979.

[15] B. Paczyński and M. Prószyński, "Models of radiation-driven winds from general relativistic neutron stars," Astrophysical Journal, vol. 302, pp. 519-524, 1986.

[16] R. Turolla, L. Nobili, and M. Calvani, "On hydrodynamics of radiatively driven winds," Astrophysical Journal, vol. 303, pp. 573-581, 1986.

[17] B. Paczyński, "Super-Eddington winds from neutron stars," Astrophysical Journal, vol. 363, no. 1, pp. 218-226, 1990.

[18] L. Nobili, R. Turolla, and I. Lapidus, "Winds from neutron stars and strong type I X-ray bursts," Astrophysical Journal, vol. 433, no. 1, pp. 276-286, 1994.

[19] J. Fukue, "Relativistic radiative flow in a luminous disk," Publications of the Astronomical Society of Japan, vol. 57, no. 6, pp. 1023-1030, 2005.
[20] J. Fukue, "Velocity-dependent eddington factor in relativistic radiative flow," Publications of the Astronomical Society of Japan, vol. 58, no. 2, pp. 461-467, 2006.

[21] J. Fukue and C. Akizuki, "Relativistic radiative flow in a luminous disk II," Publications of the Astronomical Society of Japan, vol. 58, no. 6, pp. 1073-1079, 2006.

[22] J. Fukue and C. Akizuki, "Relativistic radiation hydrodynamical accretion-disk winds," Publications of the Astronomical Society of Japan, vol. 59, no. 5, pp. 1027-1032, 2007.

[23] J. Fukue and C. Akizuki, "Spherical relativistic radiation flows with variable Eddington factor," Publications of the Astronomical Society of Japan, vol. 60, no. 2, pp. 337-343, 2008.

[24] C. Akizuki and J. Fukue, "Black-hole winds with a variable eddingtion factor," Publications of the Astronomical Society of Japan, vol. 61, no. 3, pp. 543-548, 2009.

[25] G. E. Eggum, F. V. Coroniti, and J. I. Katz, "Jet production in super-Eddington accretion disks," Astrophysical Journal, vol. 298, pp. L41-L45, 1985.

[26] G. E. Eggum, F. V. Coroniti, and J. I. Katz, "Radiation hydrodynamic calculation of super-Eddington accretion disks," Astrophysical Journal, vol. 330, pp. 142-167, 1988.

[27] W. Kley, "Radiation hydrodynamics of the boundary layer in accretion disks. II-optically thick models," Astronomy and Astrophysics, vol. 222, no. 1-2, pp. 141-149, 1989.

[28] T. Okuda, M. Fujita, and S. Sakashita, "Two-dimensional accretion disk models: inner accretion disks of $\mathrm{FU}$ orionis objects," Publications of the Astronomical Society of Japan, vol. 49, no. 6, pp. 679-697, 1997.

[29] T. Okuda, V. Teresi, E. Toscane, and D. Molteni, "Black hole accretion discs and jets at super-Eddington luminosity," Monthly Notices of the Royal Astronomical Society, vol. 357, no. 1, pp. 295-303, 2005.

[30] W. Kley and D. N. C. Lin, "Evolution of FU Orionis outbursts in protostellar disks," Astrophysical Journal, vol. 518, no. 2, pp. 833-847, 1999.

[31] T. Okuda and M. Fujita, "Super-Eddington Accretion-disk models for SS 433," Publications of the Astronomical Society of Japan, vol. 52, pp. L5-L8, 2000.

[32] T. Okuda, "Super-Eddington black-hole models for SS 433," Publications of the Astronomical Society of Japan, vol. 54, no. 2, pp. 253-266, 2002.

[33] K. Ohsuga, M. Mori, T. Nakamoto, and S. Mineshige, "Supercritical accretion flows around black holes: two-dimensional, radiation pressure-dominated disks with photon trapping," Astrophysical Journal, vol. 628, no. 1, pp. 368-381, 2005.

[34] K. Ohsuga, S. Mineshige, M. Mori, and Y. Kato, "Global radiation-magnetohydrodynamic simulations of black-hole accretion flow and outflow: unified model of three states," Publications of the Astronomical Society of Japan, vol. 61, no. 3, pp. L7-L11, 2009.

[35] K. Ohsuga, "Two-dimensional radiation-hydrodynamic model for limit-cycle oscillations of luminous accretion disks," Astrophysical Journal, vol. 640, no. 2, pp. 923-928, 2006.

[36] C. Akizuki, "The Structure of a Slim Disk Outflow Explored by Three-Dimensional Radiation Transfer," Dr. thesis at Tsukuba University, 2010.

[37] J. Fukue, "Radiative transfer in accretion-disk winds," Publications of the Astronomical Society of Japan, vol. 59, no. 3, pp. 687-694, 2007.

[38] J. Fukue, "Radiative transfer in relativistic accretion-disk winds," Publications of the Astronomical Society of Japan, vol. 60, no. 1, pp. 137-144, 2008. 
[39] J. Fukue, "Milne-Eddington solutions for relativistic planeparallel flows," Publications of the Astronomical Society of Japan, vol. 60, no. 3, pp. 627-636, 2008.

[40] J. Fukue, "Progress of Theoretical Physics," vol. 125, no. 4, pp. 837-850, 2010.

[41] J. Fukue, "Milne-eddington solutions for relativistic spherical flows," Publications of the Astronomical Society of Japan, vol. 62, no. 2, pp. 255-262, 2010.

[42] S. Kato, J. Fukue, and S. Mineshige, Black-Hole Accretion Disks, Kyoto University Press, Kyoto, Japan, 1998.

[43] S. Kato, J. Fukue, and S. Mineshige, Black-Hole Accretion Disks-Towards a New Paradigm, Kyoto University Press, Kyoto, Japan, 2008.

[44] D. Mihalas and L. H. Auer, "On laboratory-frame radiation hydrodynamics," Journal of Quantitative Spectroscopy and Radiative Transfer, vol. 71, no. 1, pp. 61-97, 2001.

[45] J. Fukue, "Relativistic variable Eddington factor," Publications of the Astronomical Society of Japan, vol. 60, no. 2, pp. 377-386, 2008.

[46] J. Fukue, "Variable Eddington factor in a relativistic planeparallel flow," Publications of the Astronomical Society of Japan, vol. 60, no. 5, pp. 1209-1216, 2008.

[47] J. Fukue, "Relativistic variable eddington factor in a relativistic plane-parallel flow," Publications of the Astronomical Society of Japan, vol. 61, no. 2, pp. 367-374, 2009.

[48] L. H. Auer and D. Mihalas, "On the use of variable Eddington factors in non-LTE stellar atmospheres computations," Monthly Notices of the Royal Astronomical Society, vol. 149, p. 65, 1970.

[49] S. M. Fu, J. Y. Wu, and X. R. Zhang, "Effects of timing acupuncture on nuclear volume of neurosecretory cells in rat supraoptic nucleus," Progress in Clinical and Biological Research, vol. 227, pp. 323-334, 1987.

[50] M.-G. Park, "Self-consistent models of spherical accretion onto black holes: II. Two temperature solutions with pairs," Astrophysical Journal, vol. 354, p. 83, 1990.

[51] A. Koerner and H. Janka, "Approximate radiative transfer by two-moment closure-when is it possible?" Astronomy and Astrophysics, vol. 266, no. 1, pp. 613-618, 1992.

[52] J. Jeans, "The stability of a spherical nebula," Philosophical Transactions of the Royal Society of London. Series A, vol. 199, pp. 1-53, 1902.

[53] E. Lifshitz, Jl. Phys. Acade. Sci. USSR, vol. 10, p. 116, 1946.

[54] C. Akizuki and J. Fukue, "Spherical relativistic radiation flows with variable Eddington factor," Publications of the Astronomical Society of Japan, vol. 60, no. 2, pp. 337-343, 2008. 

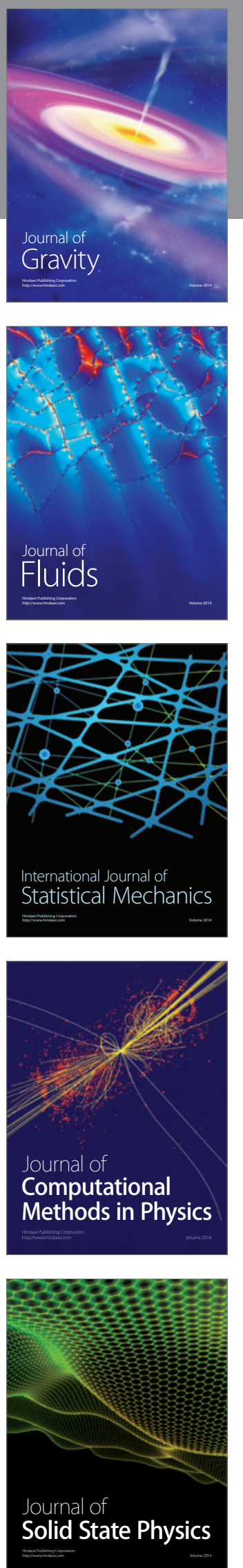

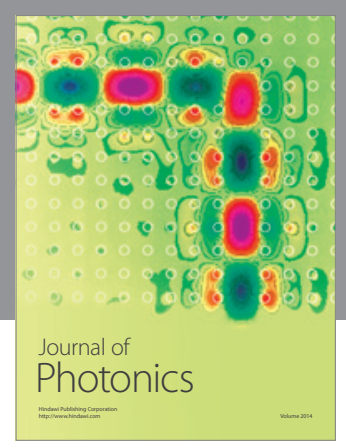

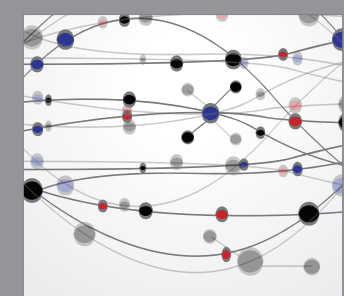

The Scientific World Journal
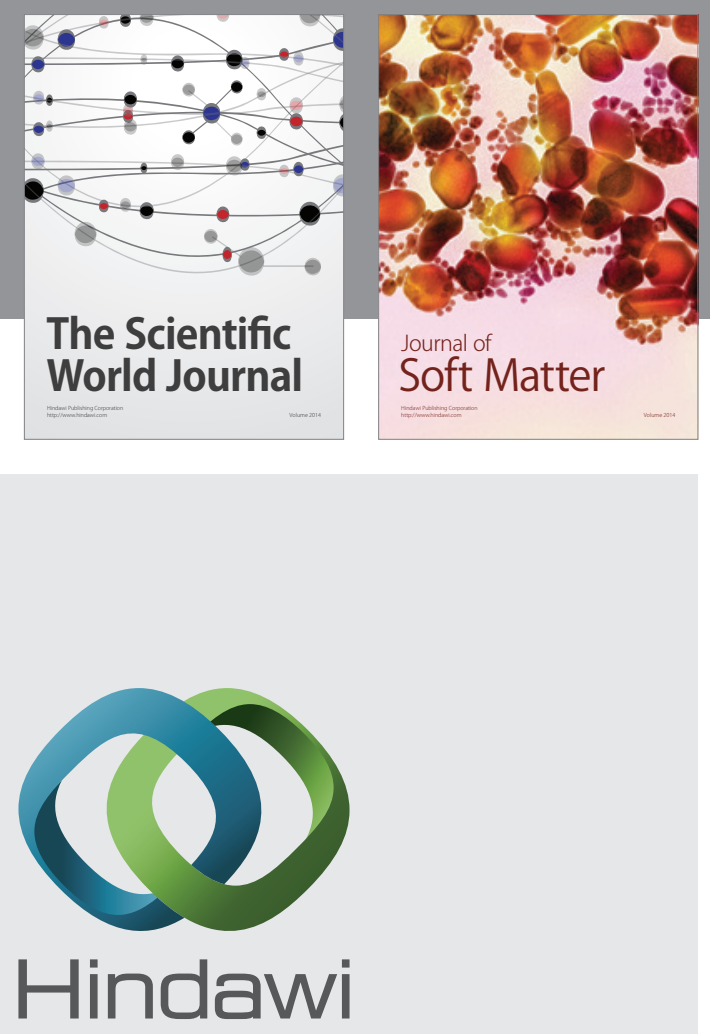

Submit your manuscripts at

http://www.hindawi.com
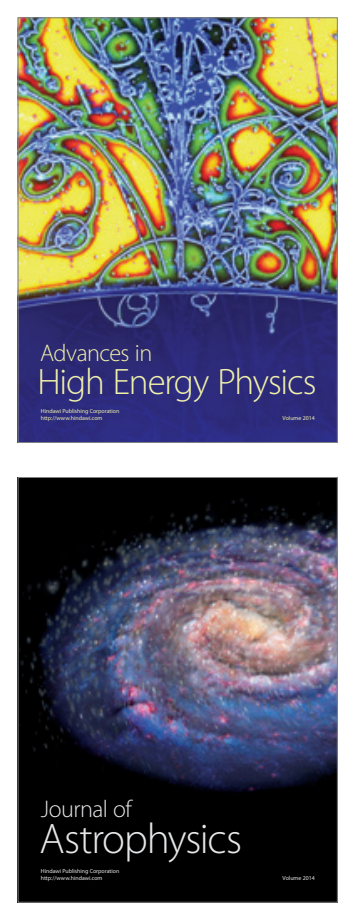
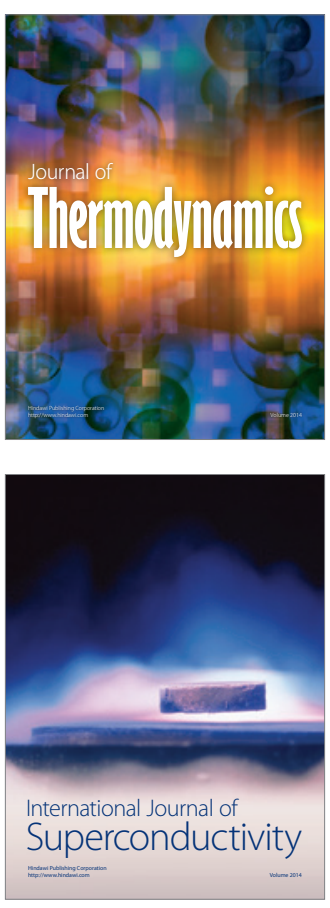
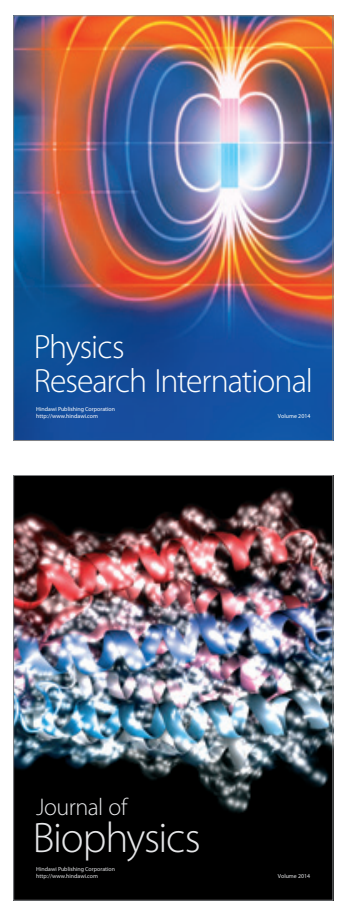
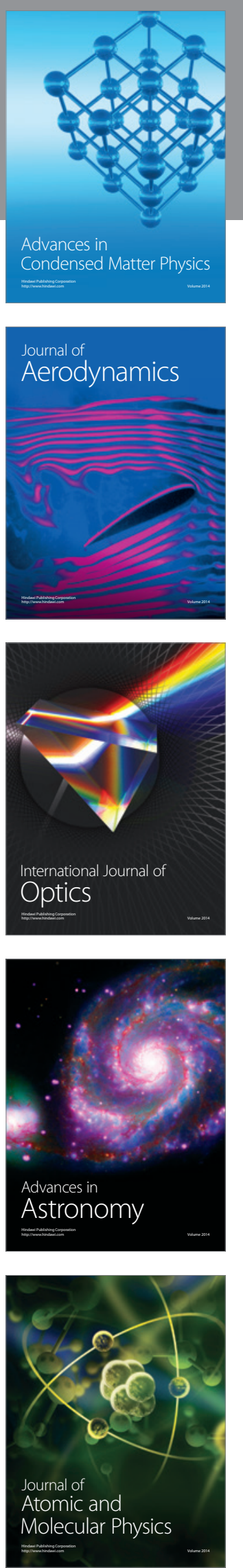